\title{
A new least square based reproducing kernel space method for solving regular and weakly singular 1D Volterra-Fredholm integral equations with smooth and nonsmooth solutions
}

\author{
Minqiang $\mathrm{Xu}^{1}$, Jing $\mathrm{Niu}^{2}$, Emran Tohidi ${ }^{3}$, and Jinjiao $\mathrm{Hou}^{4}$ \\ ${ }^{1}$ Zhejiang University of Technology \\ ${ }^{2}$ Harbin Normal University \\ ${ }^{3}$ Ton Duc Thang University \\ ${ }^{4}$ Harbin Normal University - Songbei Campus
}

August 11, 2020

\begin{abstract}
Based on the least square method, we proposed a new algorithm to obtain the solution of the second kind regular and weakly singular Volterra-Fredholm integral equations in reproducing kernel spaces. The stability and uniform convergence of the algorithm are investigated in details. Numerical experiments verify the theoretical findings. Meanwhile this method is also applicable to the nonlinear Volterra integral equations. Test problems which have non-smooth solutions are also considered and our proposed method is efficient as some recent Krylov subspace methods such as LSQR and LSMR.
\end{abstract}

\section{Hosted file}

wileyNJD-AMA.pdf available at https://authorea.com/users/350291/articles/475162-a-new-leastsquare-based-reproducing-kernel-space-method-for-solving-regular-and-weakly-singular-1dvolterra-fredholm-integral-equations-with-smooth-and-nonsmooth-solutions

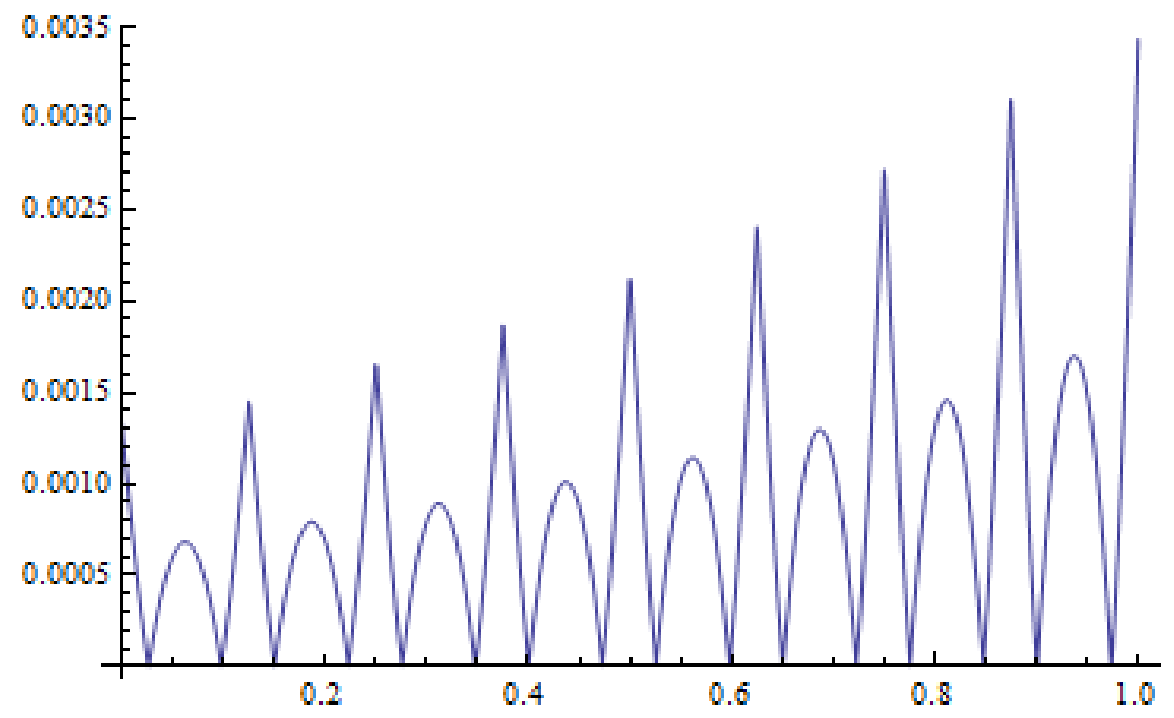



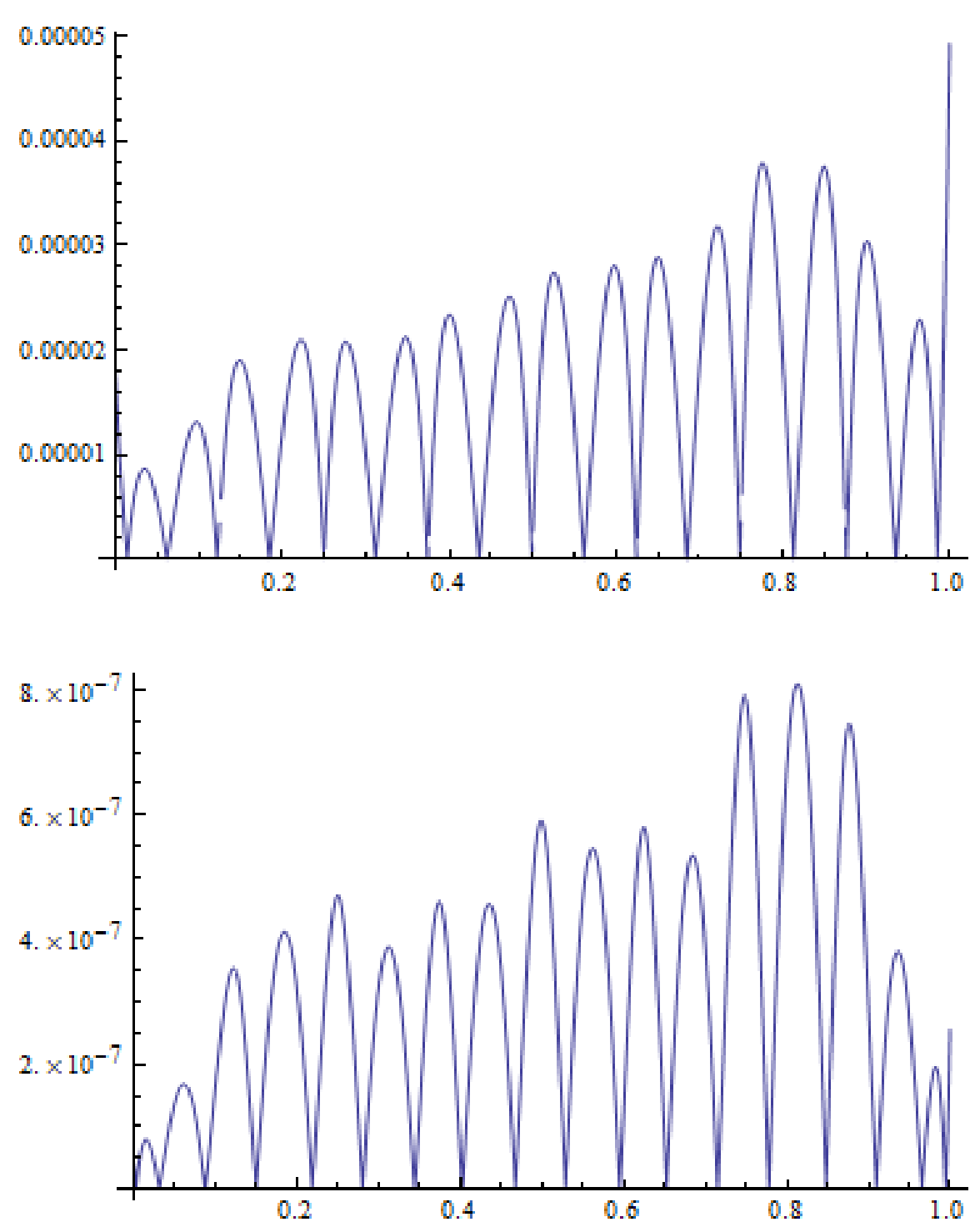

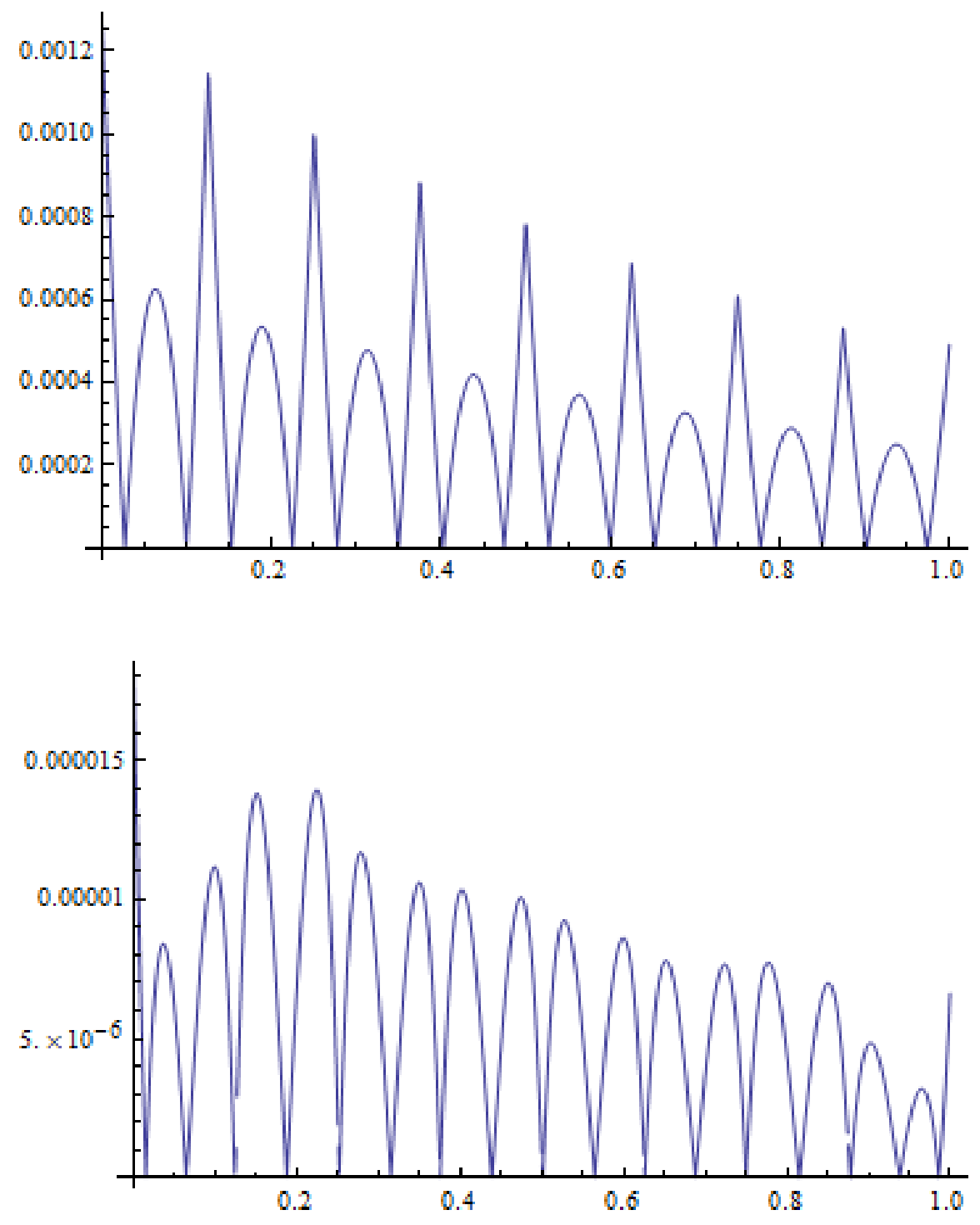

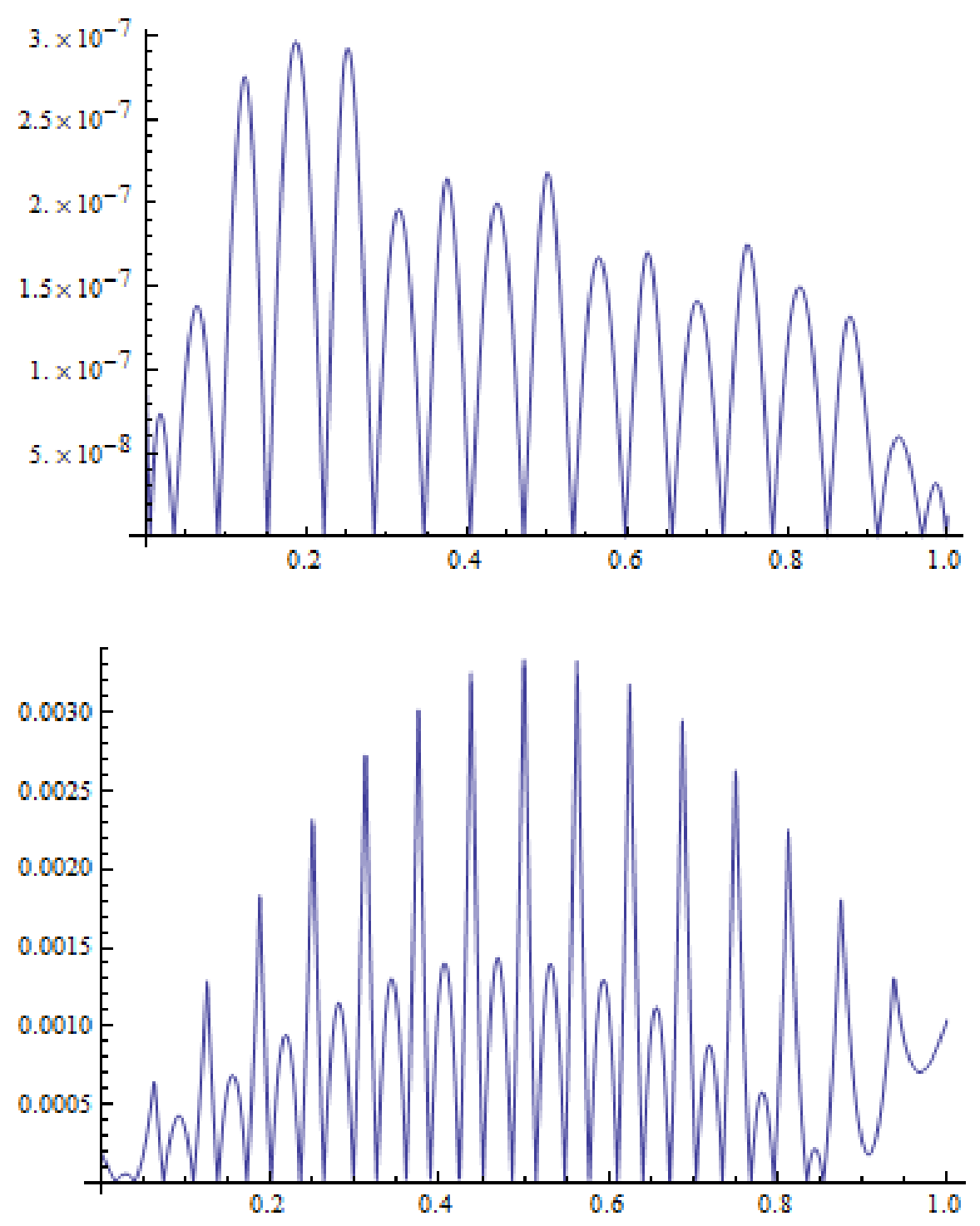

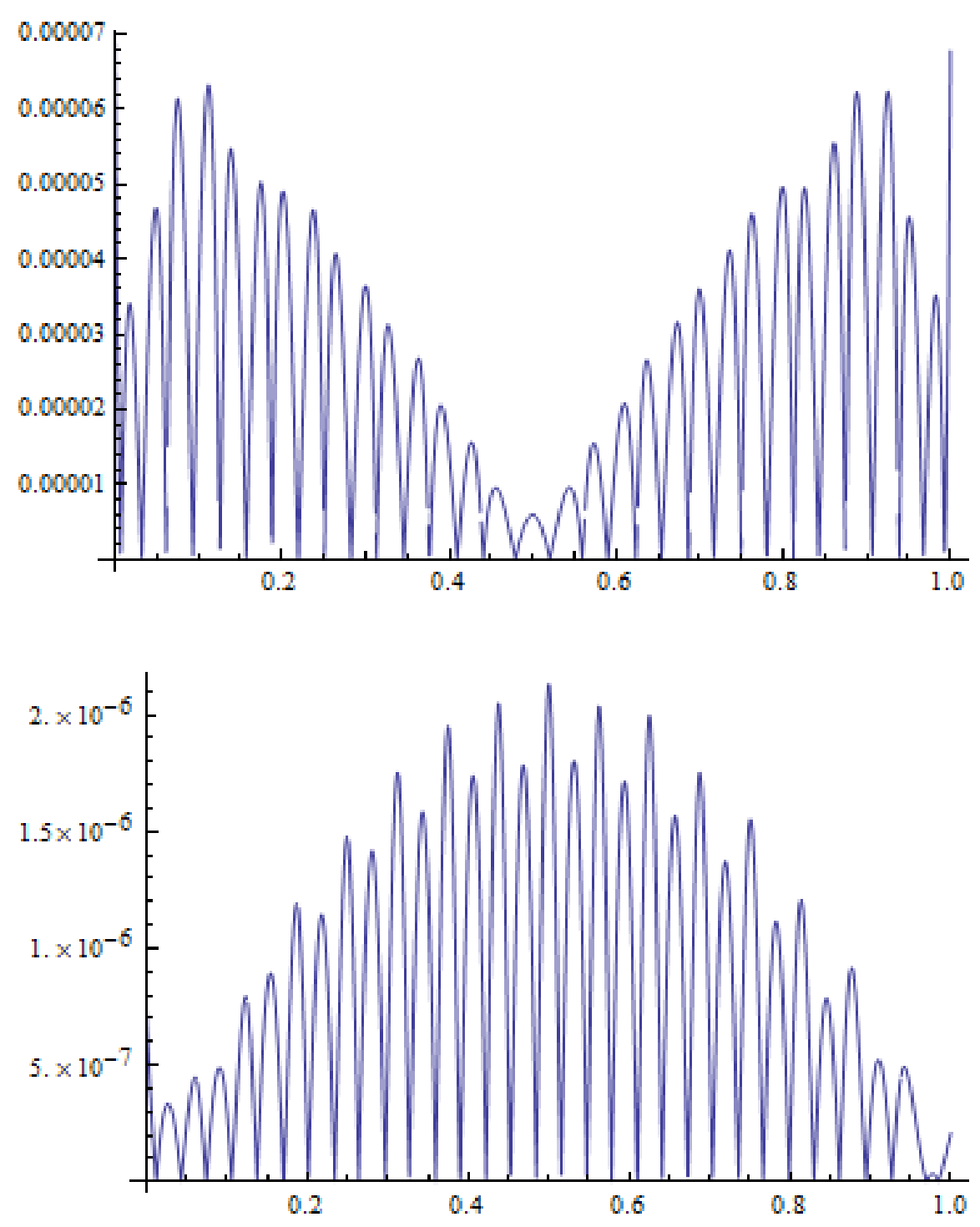

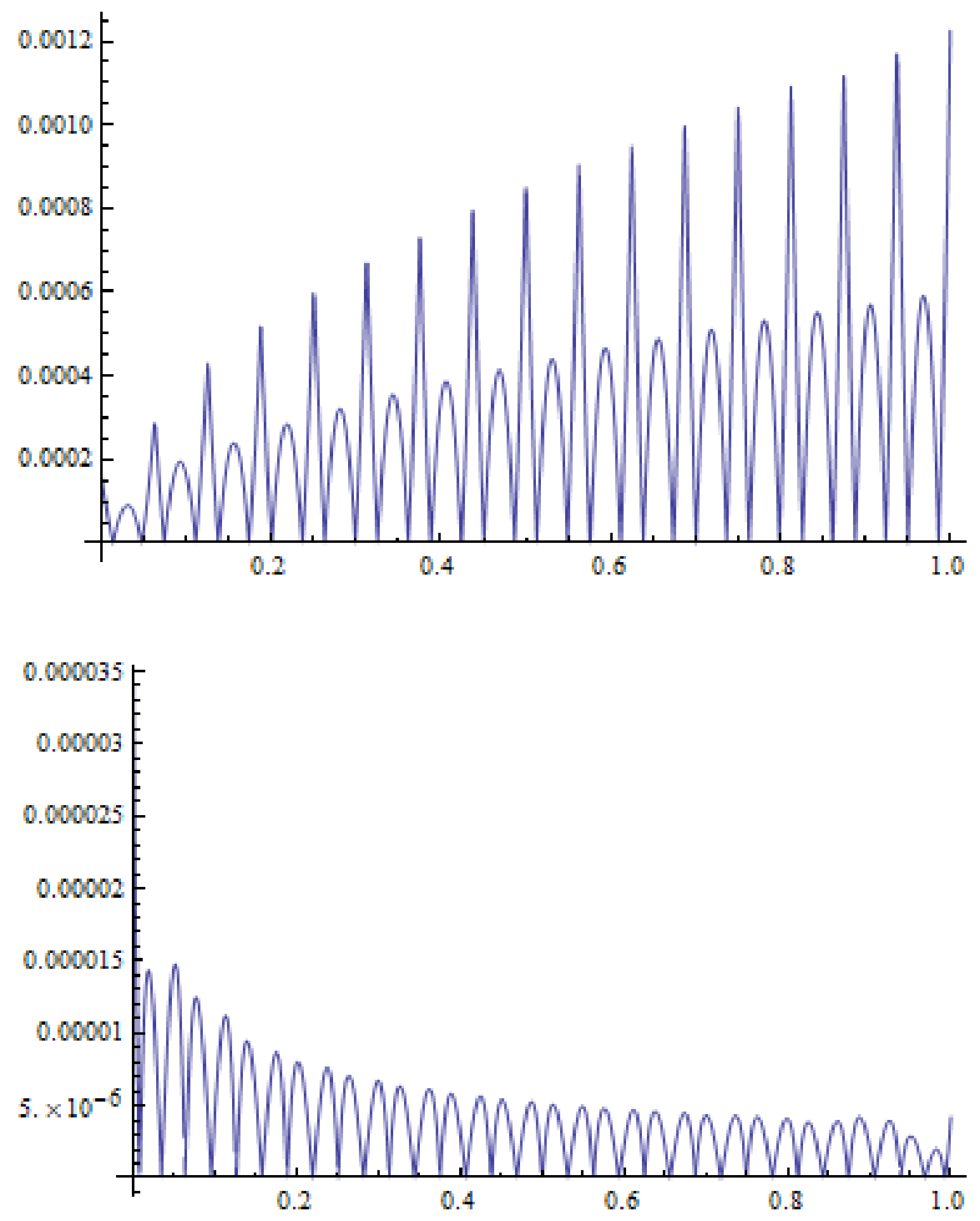

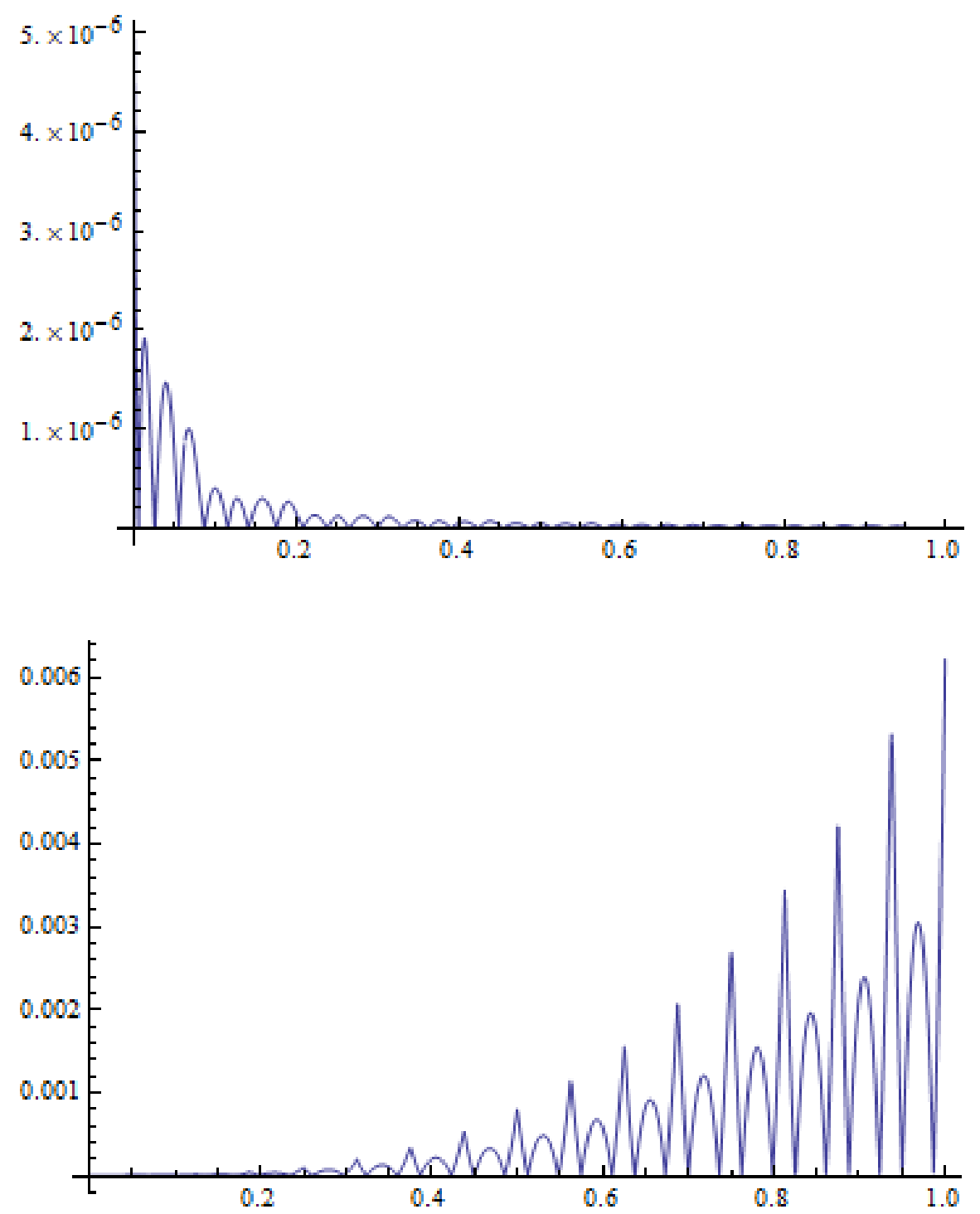

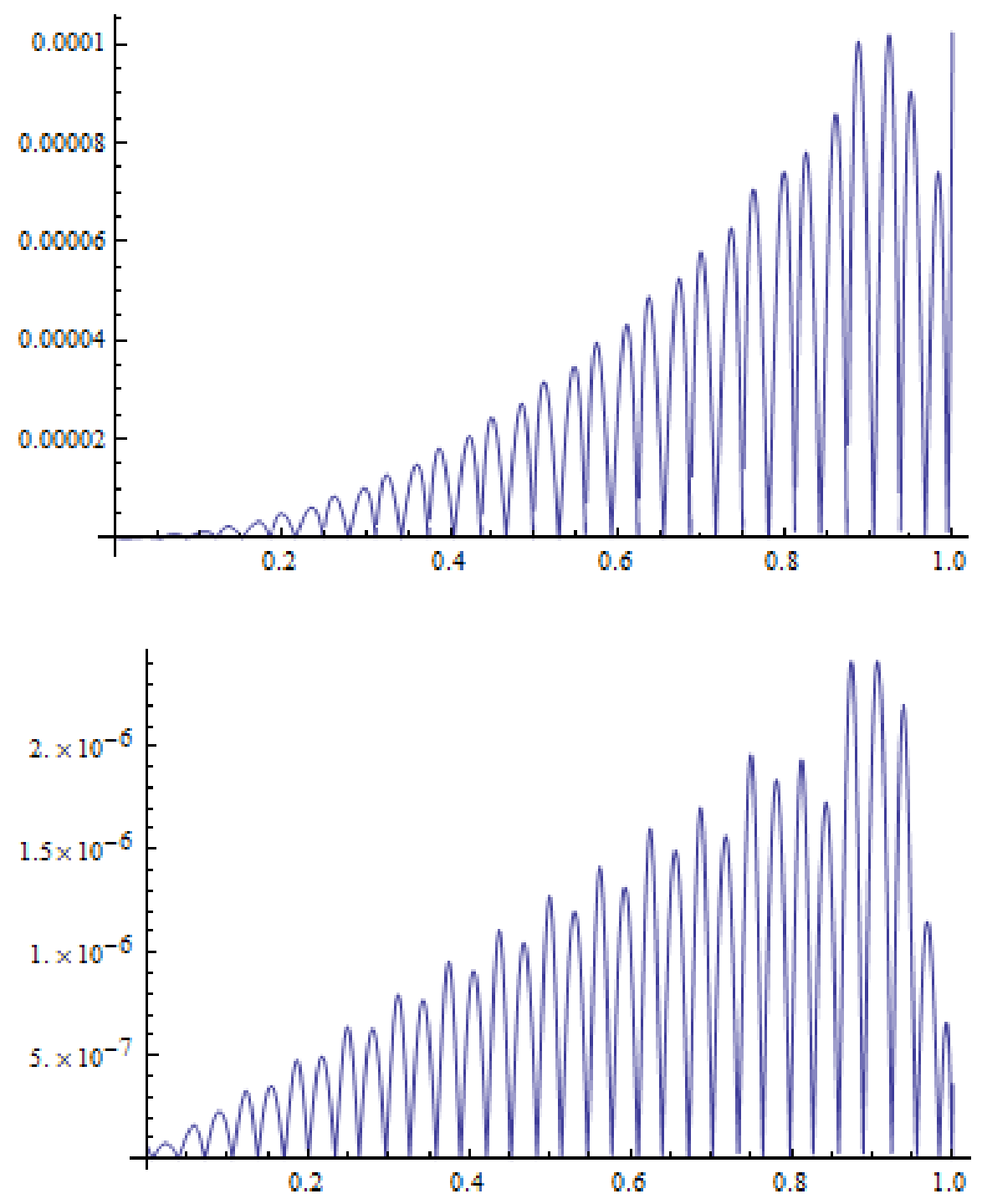

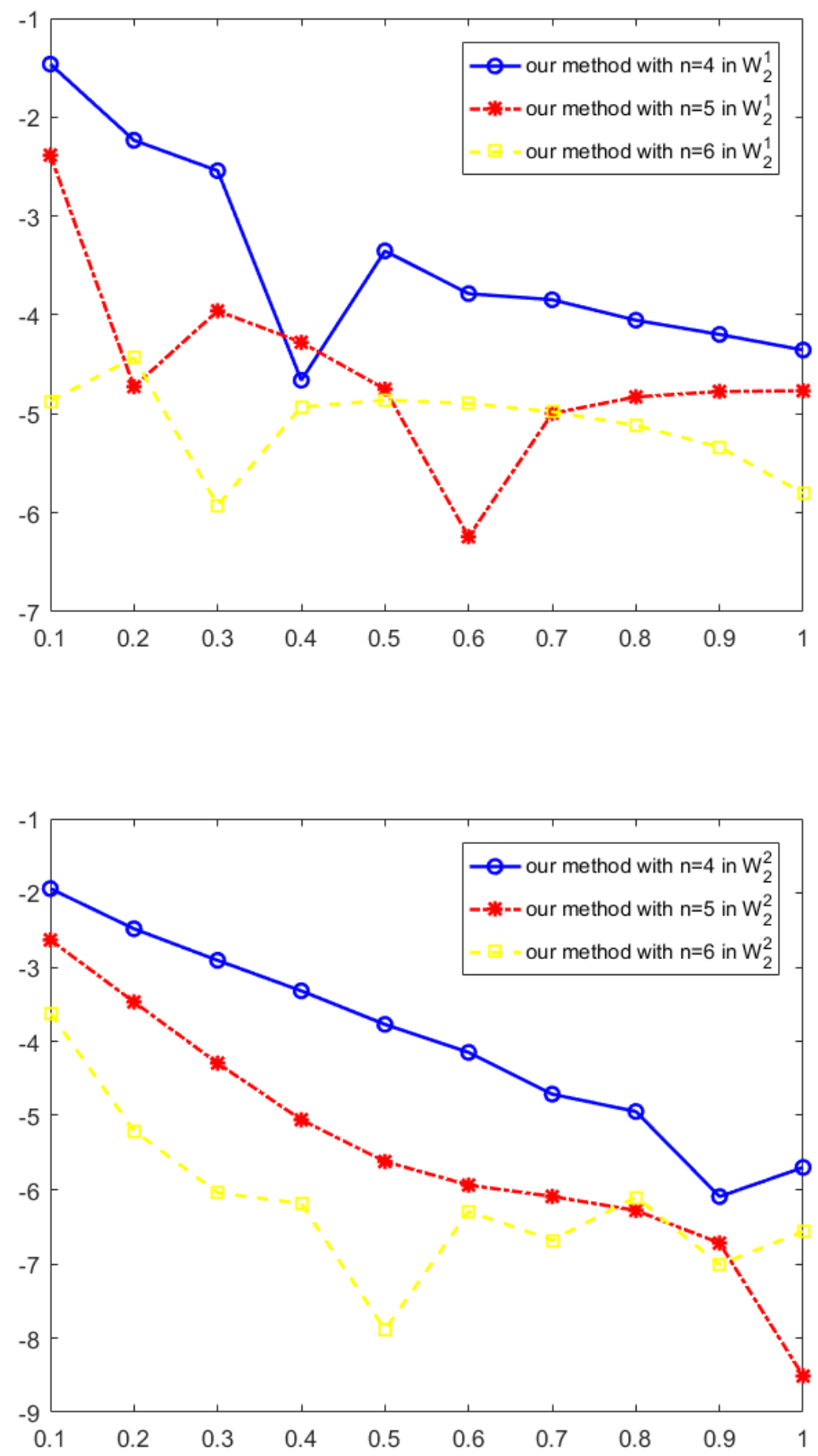


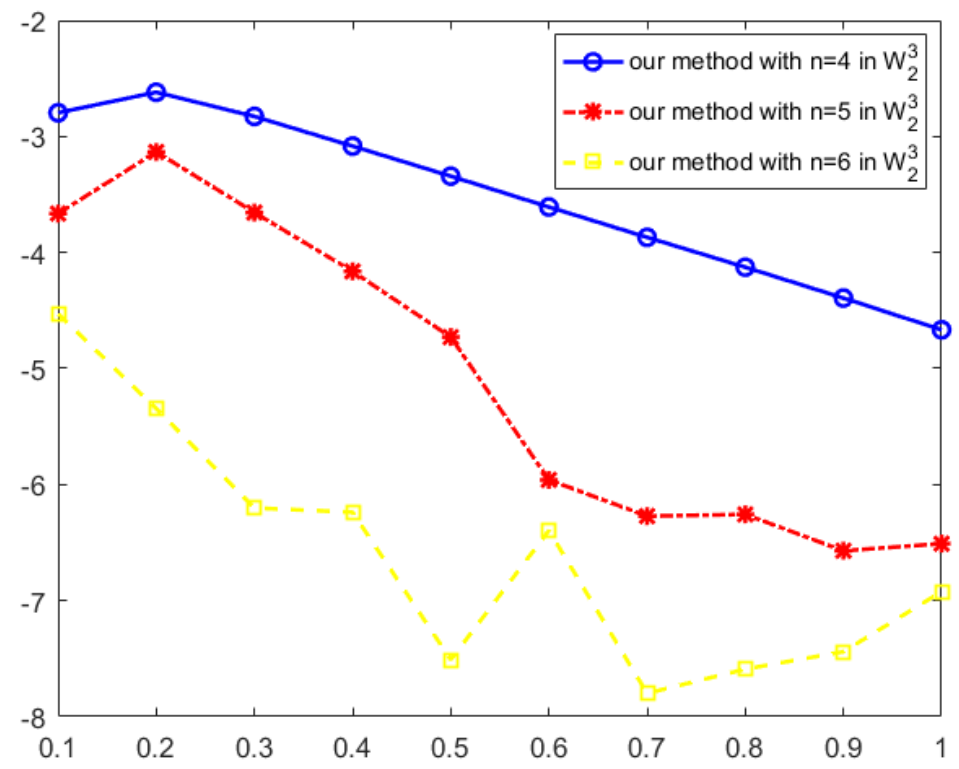

